\section{Kooperation der DGOU und der Orthopaedic Research Society (ORS)}

\author{
Der im Frühjahr 2015 geschlossene erste Kooperationsvertrag \\ zwischen der DGOU und der Orthopaedic Research Society \\ (ORS) hatte das Ziel, die Internationalisierung der DGOU weiter \\ voranzutreiben. Die Aktivitäten des letzten Jahres und die hier- \\ aus entstandenen Folgeprojekte belegen, dass es hierdurch \\ tatsächlich zu einer erheblichen Vertiefung der Zusammenar- \\ beit beider Gesellschaften gekommen ist. Mit den folgenden \\ Ausführungen sollen wesentliche Aspekte der Kooperation vor- \\ gestellt werden.
}

Im Rahmen des DKOU 2015 wurden vier grundlagenwissenschaftliche Sitzungen in Kooperation mit der ORS gestaltet. In jeder dieser englischsprachigen Veranstaltungen wurde durch einen ausgewiesenen Experten der ORS ein Hauptvortrag gehalten. Aufgrund des großen Besucherzuspruchs und des hervorragenden wissenschaftlichen Austausches mit den Referenten der ORS war die Etablierung dieser Sitzungen ein großer Erfolg. Zur Honorierung dieser Zusammenarbeit wurde Deutschland im Gegenzug als Gastland des Jahreskongresses 2016 der ORS in Orlando ausgewählt. Im Rahmen dieses Kongresses konnte die Preisträgerin 2015 des Grundlagenforschungspreises der DGOU, Dr. Tanja Niedermaier, in der „ORS excellence in orthopaedics awards session“ ihre diesem Preis zu Grunde liegenden Forschungsergebnisse präsentieren. Ebenso wurden Prof. Dr. Heiko Reichel und Prof. Dr. Florian Gebhard als diesjährige Präsidenten der DGOU, Prof. Dr. Michael Nerlich als Präsident der DGOU 2015 und Initiator der Kooperation sowie Prof. Dr. Frank Hildebrand als Präsident der Sektion Grundlagenforschung zu dem Jahreskongress eingeladen. In einem gemeinsamen Treffen mit dem Präsidium der ORS wurden weitere Kooperationsmöglichkeiten abgestimmt ( 0 Abb. 1).

\section{Erfolgreiche Kooperation $\nabla$}

So wurde in dieser Zusammenkunft aufgrund der äußerst erfolgreichen Zusammenarbeit beschlossen, die Kooperation langfristig fortzuführen. Hierzu wurde ein dreijähriger Kooperationsvertrag ausgearbeitet, der insbesondere die Koordination gemeinsamer Aktivitäten beinhaltet. So wurde zur zusätzlichen Förderung des wissenschaftlichen Austauschs zwischen der DGOU und der ORS auf Initiative der diesjährigen Präsidenten der DGOU die Vergabe eines ORS Travel Awards beschlossen. Die Sektion Grundlagenforschung wurde mit der Erstellung von Vergabekriterien des mit 3.000 Euro dotierten Preises beauftragt. Die Verleihung erfolgt erstmalig in einer Preisträgersitzung im Rahmen der DKOU 2016, deren Teilnehmer auf Basis der Gutachterbewertung der eingereichten Abstracts ermittelt wurden. Zukünftig ist eine aktive Bewerbung für diesen Preis möglich.

Zusätzlich wurden für den DKOU 2016 erneut vier Sitzungen in Kooperation mit der ORS vereinbart, in denen ausgewiesene Experten der ORS Keynote Vorträge halten werden. Dieses sind im Einzelnen:

Prof. Dr. Thomas Andriacci ist ehemaliges Mitglied des „Board of Directors“ der ORS und trägt als aktives Mitglied der ORS regelmäßig als Gutachter und Vorsitzender zu erfolgreichen Kongressen bei. Als emeritierter Professor am Department of Mechanical Engineering der School of Engineering und am Department of Orthopaedic Surgery der School of Medicine der Stanford University gilt sein besonderes Interesse der Behandlung der Folgen von Arthrose, Sportverletzungen und neuromuskulären Erkrankungen. Er arbeitet weiterhin aktiv an der Entwicklung eines interdisziplinären Konzepts, um die Interaktionen von biologischen, strukturellen und funktionellen Einflussgrößen auf die Entstehung und Behandlung der Gonarthrose zu untersuchen.

Dr. Alison Gartland ist aktives Mitglied der ORS und gestaltet regelmäßig als Gutachter und Vorsitzender die Kongresse der ORS mit. Am Department of Oncology \& Metabolism der Universität Sheffield be-

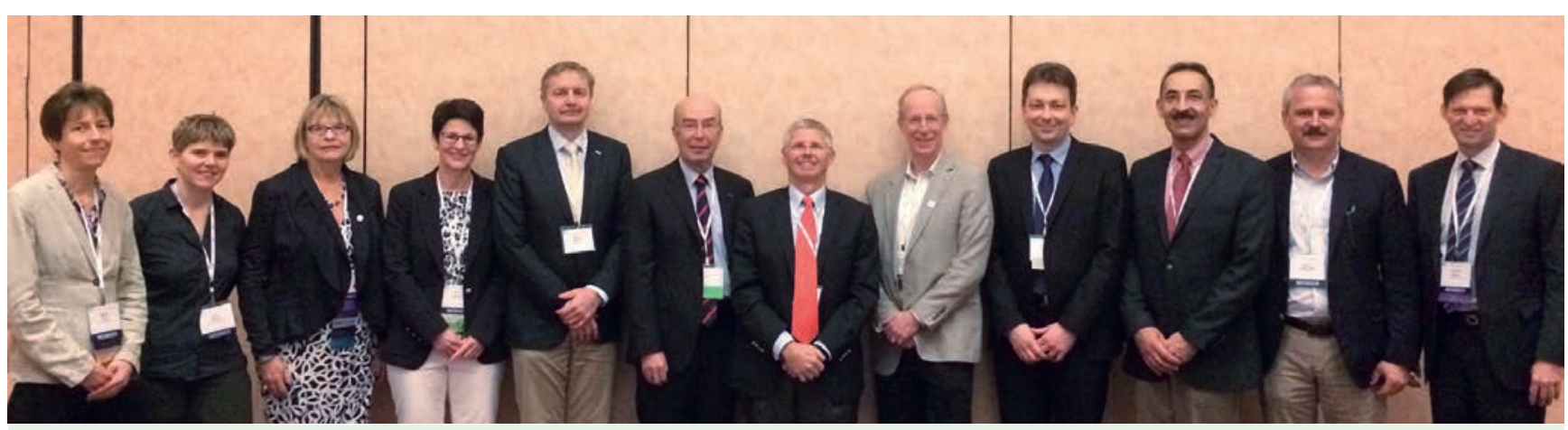

Abb. 1 Sibylle Grad, Alison Gartland, Mary Goldring, Anita Ignatius, Heiko Reichel, Michael Nerlich, Mathias Bostrom, Rick Sumner, Frank Hildebrand, Farshid Guilak, Ted Miclau (v. I. n. r.). Bild: DGOU 


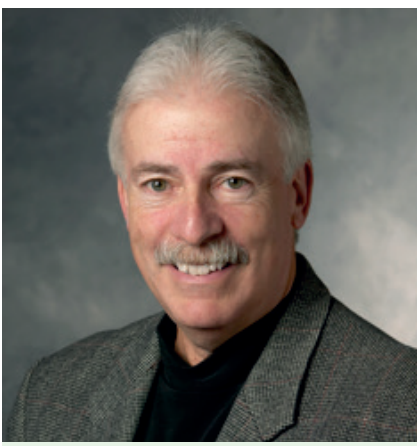

Thomas Andriacchi, PhD Professor

Department of Mechanical Engineering, School of Engineering Stanford University

Professor

Department of Orthopaedic Surgery, School of Medicine Stanford University

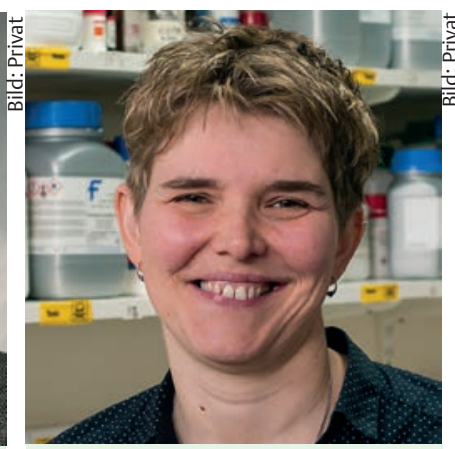

Alison Gartland, PhD

Lecturer in Bone and Cancer Biology

Academic Unit of Bone Biology Department of Oncology \& Metabolism

University of Sheffield Medical School

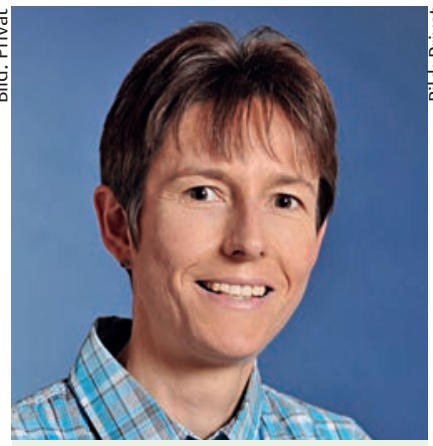

Sibylle Grad, PhD

Principal Scientist \& Deputy Program Leader

Musculoskeletal Regeneration Program

AO Research Institute Davos

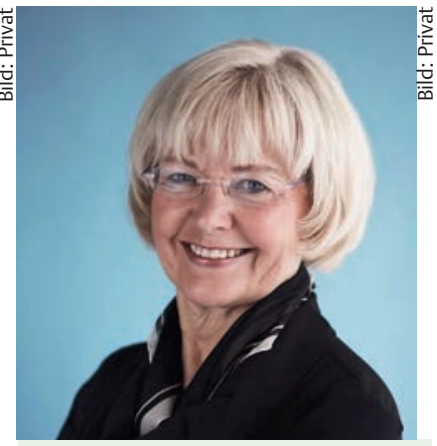

Linda Sandell, $P h D$

Director

Core Centers for Musculoskeletal

Biology and Medicine

Washington University

Mildred B. Simon Professor

Department of Orthopaedic Surgery, Washington University

Editor in Chief

Journal of Orthopaedic Research schäftigt sie sich vor allem mit der Knochen- und Tumorbiologie sowie mit muskuloskelettalen Erkrankungen im Rahmen maligner Grunderkrankungen. In aktuellen Projekten untersucht sie Aspekte zur ossären Metastasierung bei Mammakarzinom und zur Bedeutung von purinergen Rezeptoren im Knochen.

Dr. Sibylle Grad ist aktives Mitglied der ORS und arbeitet regelmäßig als Gutachter, Vorsitzender und Research Interest Group (RIG) Organizer bei der Organisation der Kongresse der ORS mit. Als Principal Scientist und stellvertretender Program Leader der Forschungsgruppe "Musculoskeletal Regeneration" am AO Forschungsinstitut in Davos gilt ihr besonderes Interesse der Bandscheiben- und Knorpelregeneration. Hierbei stehen insbesondere neue zellbasierte und molekulare Therapien im Fokus. Dr. Grad sieht den Schlüssel zum Erfolg für neue biologische Therapien vor allem in der engen $\mathrm{Zu}$ sammenarbeit von Grundlagenforschern und klinisch tätigen Ärzten.

Prof. Dr. Linda Sandell ist ehemalige Präsidentin der ORS und Gründerin des Women's Leadership Forum. Als Mildred B. Simon Professor am Department of Orthopaedic Surgery und als Direktor des Core Centers for Musculoskeletal Biology and Medicine der Washington University gehört sie zu den Pionieren der molekularbiologischen Analysen in der orthopädischen Forschung, um die zellulären Vorgänge der Knorpelverletzung und Arthro- se zu untersuchen. Dafür wurde sie 2016 unter anderem mit dem "Distinguished Investigator Award" der ORS ausgezeichnet.

Zusammenfassend lässt sich feststellen, dass mit den geschilderten Aktivitäten eine hervorragende Basis für die Zusammenarbeit der DGOU und der ORS geschaffen wurde, die mit dem nun abgeschlossenen langfristigen Kooperationsvertrag auch zukünftig zu einer Internationalisierung der DGOU beitragen wird.

\section{Sitzungstermine mit ORS-Beteili- gung im Rahmen des DKOU 2016 $\nabla$}

\section{Sitzungsnummer: GR12}

Sitzungstitel: Travel Award ORS

Sitzungszeit: Dienstag, 25.10.2016, 11:00

- 12:30 Uhr

Ort: Berlin 2

Vortragstitel: ORS KEYNOTE: Joint Physiology \& Mechanics: Implications for Osteoarthritis

Vortragende: Andriacchi Thomas (Stanford, USA)

\section{Sitzungsnummer: GR14}

Sitzungstitel: Stem cells and tissue repair Sitzungszeit: Dienstag, 25.10.2016, 16:30 - 18:00 Uhr

\section{Ort: Berlin 2}

Vortragstitel: ORS KEYNOTE: Stem cells for intervertebral disc regeneration: success and hurdles

Vortragende: Grad Sibylle (Davos, $\mathrm{CH}$ )

\section{Sitzungsnummer: GR16}

Sitzungstitel: Osteoarthritis and cartilage Sitzungszeit: Mittwoch, 26.10.2016, 11:00 - 12:30 Uhr

Ort: Berlin 2

Vortragstitel: ORS KEYNOTE: Joint Injury \& Osteoarthritis GR16-2002

Vortragende: Sandell Linda (St. Louis, USA)

\section{Sitzungsnummer: GR18}

Sitzungstitel: Bone biology and repair

Sitzungszeit: Mittwoch, 26.10.2016, 16:30 - 18:00 Uhr

Ort: Berlin 2

Vortragstitel: ORS KEYNOTE: Bone Biology and Repair

Vortragende: Gartland Allie (Sheffield, UK)

Hildebrand $F^{1}$, Gebhard $F^{2}$, Reichel $H^{3}$, Nerlich $M^{4}$, Loibl $M^{4}$

1 Klinik für Unfall- und Wiederherstellungschirurgie, Universitätsklinikum Aachen

2 Klinik für Unfall-, Hand-, Plastische- und Wiederherstellungschirurgie, Universitätsklinikum Ulm

3 Ärztlicher Direktor, Orthopädische Universitätsklinik, Universitäts- und Rehabilitationskliniken Ulm

4 Klinik und Poliklinik für Unfallchirurgie, Universitätsklinikum Regensburg

Dieser Bericht erscheint zeitgleich in der Orthopädie und Unfallchirurgie Mitteilungen und Nachrichten. 